\title{
UMA AVALIAÇÃO DAS PREVISÕES DO MODELO REGIONAL ETA EM ALTA RESOLUÇÃO PARA DOIS CASOS DE CHUVA INTENSA OCORRIDOS NA REGIÃO DA SERRA DO MAR.
}

\author{
FERNANDA CERQUEIRA VASCONCELLOS, IRACEMA FONSECA DE ALBUQUERQUE \\ CAVALCANTI
}

\author{
Instituto Nacional de Pesquisas Espaciais, Centro de Previsão de Tempo e Estudos Climáticos/Instituto \\ Nacional de Pesquisas Espaciais (CPTEC/INPE)
}

fernanda.cerqueira@cptec.inpe.br, iracema.cavalcanti@cptec.inpe.br

Recebido Setembro 2009 - Aceito Março 2010

\begin{abstract}
RESUMO
As chuvas intensas na Região Sudeste freqüentemente causam enchentes e deslizamentos, principalmente nas proximidades da Serra do Mar. Por esta razão, o estudo de eventos intensos nesta região é importante para entender o comportamento dos mesmos e melhorar suas representações em modelos numéricos de previsão. Dois casos de chuva intensa foram escolhidos para este estudo (02/02/1983 e 25/05/2005). Em ambos os casos houve ocorrência de um aglomerado convectivo embebido em um sistema frontal. No primeiro caso a situação sinótica possuía configurações de um sistema tipo $\mathrm{Y}$, cuja principal característica é a presença de nebulosidade sobre a América do Sul de forma semelhante a um "Y". Essa nebulosidade está relacionada a três sistemas: um sistema frontal, Alta da Bolívia e um VCAN próximo ao nordeste brasileiro. No segundo caso havia linhas de instabilidade junto ao sistema frontal. Em ambos os casos, o Modelo Eta de alta resolução (com espaçamentos horizontais de grade de 5 e $10 \mathrm{Km}$ ) simulou razoavelmente bem as características da atmosfera na região, assim como um máximo de precipitação, embora apresentando ligeiros erros de fase quanto ao posicionamento da banda de precipitação mais intensa. Características de mesoescala puderam ser analisadas nos resultados do modelo, tais como a ação da brisa marítima (primeiro caso) e orografia (primeiro e segundo caso), cuja atuação contribuiu para a intensa precipitação causada pela ação dos sistemas sinóticos presentes. Valores altos de índices de instabilidade também foram obtidos dos resultados do modelo para os dois casos, indicando um bom desempenho do mesmo para alertas de intensa precipitação na região.

Palavras chaves: Precipitação, Serra do Mar, Modelo Regional Eta, Índices de Instabilidade.
\end{abstract}

\begin{abstract}
EVALUATION OF THE ETA REGIONAL MODEL FORECASTS FOR TWO CASES OF INTENSE RAINFALL OVER THE SERRA DO MAR REGION

Intense rainfall over Southeastern Brazil frequently causes flooding and landslides, mainly in Serra do Mar region. For this reason, the study of significant rainfall events over this region is important to understand their behavior and improve their representation in numerical prediction models. Two cases of intense rainfall were chosen for this study $(02 / 02 / 1983$ and 25/05/2005). Both cases were associated with a convective cluster embedded in a frontal system. The synoptic analysis depicted, in the first case, a type $Y$ configuration, consisting of a frontal system and cloudiness associated with the Bolivian High and the Upper Level Cyclonic Vortex over Northeastern Brazil. The second case consisted of squall lines associated with a frontal system. Despite some slight phase errors regarding the location of the most significant rainfall sector, the atmospheric pattern associated with the extreme rainfall cases over Serra do Mar were well simulated by the regional eta model, including the magnitude of the intense precipitation. Mesoscale features were analyzed in the high resolution model outputs, such as the sea breeze (first case) and orography (first and second case), which contributed to the significant rainfall near the coast in both cases. High values of instability indexes were also obtained from the model results indicating a good performance for intense precipitation alert.
\end{abstract}

Keywords: Precipitation, Serra do Mar, Eta Model, Instability Indeces. 


\section{INTRODUÇÃO}

Chuvas intensas na Região Sudeste do Brasil freqüentemente causam enchentes, deslizamentos de terra, perdas na agricultura, destruição de casas, de rodovias, de redes de energia e comunicação, principalmente nas regiões próximas à Serra do Mar e nas suas encostas. A modelagem numérica tem sido utilizada em diversos estudos de casos extremos na Região Sudeste, principalmente na região da Serra do Mar. Guimarães et al. (2007), avaliando a previsão de 48h do Modelo Regional Eta com espaçamentos horizontais de grade de 40 e $10 \mathrm{Km}$ para três casos de chuva intensa no Estado do Rio de Janeiro, mostraram que o modelo com espaçamento de $40 \mathrm{Km}$ representou bem as características em escala sinótica, incluindo o padrão geral da precipitação ao longo do período estudado. A versão de alta resolução espacial do modelo (espaçamento de $10 \mathrm{Km}$ ), também proporcionou bons resultados na previsão dos eventos locais, incluindo a representação de índices de instabilidade. Rozante e Cavalcanti (2006) estudaram a influência da condição inicial (CI) no modelo Regional MesoEta para a previsão de chuvas extremas nas proximidades da Serra do Mar. Eles mostraram que a CI perturbada do NCEP forneceu informações suficientes para o modelo representar a destabilização da atmosfera na região de ocorrência do episódio, tais como aquecimento da baixa troposfera, umidade, intensidade e direção do vento.

Os objetivos deste trabalho são analisar as influências da escala sinótica e da possível contribuição dos mecanismos de mesoescala, nos eventos extremos de precipitação ocorridos nos dias 02 de fevereiro de 1983 e 25 de maio de 2005 na Região da Serra do Mar, e avaliar a capacidade do Modelo Regional Eta em prever precipitações intensas nessa região. Segundo Vasconcellos (2008), que realizou uma climatologia da precipitação sobre parte da Região Sudeste do Brasil entre os anos de 1980 e 2006, esses casos ocorreram em anos considerados chuvosos.

\section{MATERIAL E MÉTODO}

Para a realização deste trabalho foram utilizados dados de precipitação diários analisados em pontos de grade com resolução de $1^{\circ}$ de lat/lon obtidos do NCEP (Silva et al., 2007 e Shi et al., 2000); dados diários da Reanálise do NCEP/NCAR com resolução de 2,5 de lat/lon (Kalnay et al. 1996); imagens do satélite GOES-5 E GOES-12 nos canais infravermelho e infravermelho realçada; imagens CAPPI $3 \mathrm{Km}$ do radar de São Roque (para o segundo caso) e as saídas numéricas do modelo regional Eta não-hidrostático em uma grade regular horizontal de $5 \mathrm{Km}$ (primeiro caso) e $10 \mathrm{Km}$ (segundo caso) e freqüência horária, para a avaliação do evento.

A região de interesse para o estudo é o Sudeste do Brasil, com ênfase na Região da Serra do Mar. Para a escolha dos casos, foram analisados, com dados diários de precipitação observada em pontos de grade, dias com chuva igual ou superior a $100 \mathrm{~mm} /$ dia em pelo menos um ponto de grade da região de estudo próxima à Serra do Mar. Dentre os casos que seguiram esse critério, foram escolhidos os dias 02/02/1983 e $25 / 05 / 2005$. As CIs utilizadas no modelo Eta foram obtidas da Reanálise do NCEP/NCAR (primeiro caso) e das análises do Modelo de Circulação Global Atmosférico (MCGA) do NCEP com T254L64, ou seja, com espaçamento horizontal de aproximadamente $65 \mathrm{Km}$ (segundo caso). A utilização das CIs da Reanálise do NCEP/NCAR no primeiro caso deveu-se a não disponibilidade das análises do MCGA do NCEP no período em que este caso ocorreu. A utilização das análises do MCGA do NCEP, em vez da Reanálise do NCEP/NCAR, no segundo caso fornece resultados mais próximos de uma previsão. A parametrização de convecção utilizada no modelo foi a de Kain-Fritsch (Kain e Fritsch, 1993). Detalhes do modelo podem ser encontrados em Mesinger (1984), Mesinger et al. (1988) e Chou (1996). O estudo das características da condição sinótica reinante foi realizado através dos dados de diversas variáveis da Reanálise do NCEP/NCAR. As imagens de satélite foram também utilizadas para a identificação e acompanhamento dos sistemas sinóticos atuantes. A avaliação das simulações do modelo Eta foi conduzida analisando-se as soluções numéricas em comparação com as condições sinóticas e de mesoescala observadas durante os eventos ao longo dos períodos de $24 \mathrm{~h}$ de integração do modelo. Para avaliar a simulação das condições de instabilidade atmosférica na região, foram calculados os seguintes índices de instabilidade: K (Equação 1), CK (Equação 2) e CITT (Equação 3). Em geral um valor crítico para o índice $\mathrm{K}$ no Brasil é de aproximadamente $30^{\circ} \mathrm{C}$, e quanto maior o valor de $\mathrm{K}$, maior a instabilidade (no caso de tempestades causadoras de altos índices de precipitação, como nos casos estudados neste trabalho). O CK e o CITT são índices gerados a partir do produto entre a convergência dos ventos em baixos níveis e os índices $\mathrm{K}$ e Índice Total Totals (ITT), respectivamente. Regiões favoráveis à formação de tempestades são aquelas onde a concentração de umidade apresentar-se alta, e onde há convergência em baixos níveis e valores altos dos índices K e ITT (valores altos em módulo, pois eles são negativos). Os índices CK e CITT foram definidos por Lima (2005) e Cerqueira (2006), respectivamente.

$$
\begin{aligned}
\mathrm{K} & =\mathrm{T}_{850}-\mathrm{T}_{500}+\mathrm{Td}_{850}-\mathrm{DEP}_{700} \quad\left[{ }^{\circ} \mathrm{C}\right] \\
\mathrm{CK} & =\operatorname{conv} \times \mathrm{K}=\operatorname{conv} \times(\mathrm{T} 850-\mathrm{T} 500+\mathrm{Td} 850-\mathrm{DEP} 700) \\
{\left[{ }^{\circ} \mathrm{C} . \mathrm{s}-1\right] } &
\end{aligned}
$$
$\left[{ }^{\circ} \mathrm{C} . \mathrm{s}-1\right]$

$$
\text { CITT }=\text { conv } x \text { ITT }=\text { conv } x(\text { T850 }+ \text { Td } 850-2 * \text { T500 })
$$


onde T é a temperatura do ar, Td é a temperatura do ponto de orvalho, DEP é a depressão do bulbo úmido (temperatura do ar menos temperatura do bulbo úmido - em ${ }^{\circ} \mathrm{C}$ ), conv é a convergência de massa em $900 \mathrm{hPa}$ (somente os valores negativos de $\vec{\nabla} \cdot \vec{V}$ ).

\section{RESULTADOS E DISCUSSÃO}

\subsection{Caso 02 de Fevereiro de 1983}

A Figura 1 apresenta a precipitação acumulada entre os dias $01 / 02 / 1983$ às $1200 \mathrm{Z}$ e 02/02/1983 às 1200Z. Observa-se

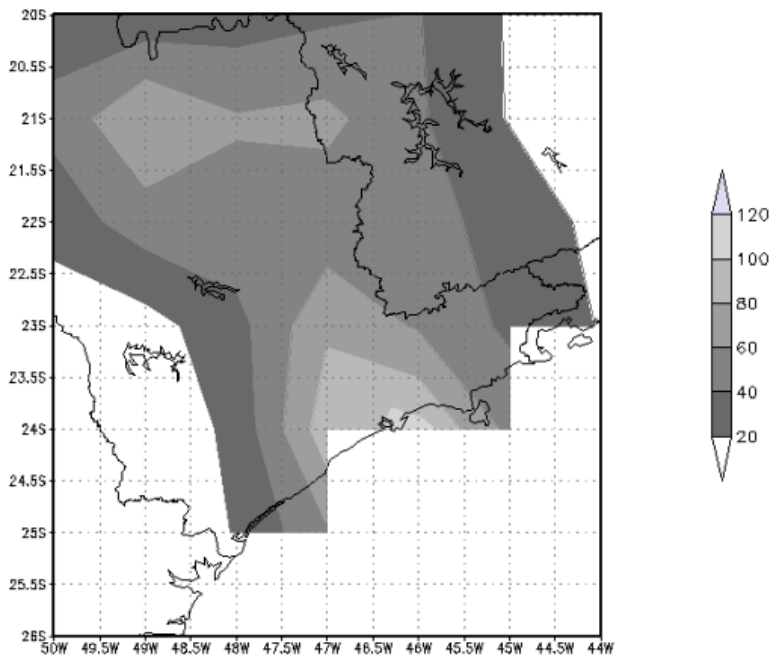

Figura 1 - Precipitação acumulada em 24h para o dia 02/02/1983. [Dados observados, em pontos de grade]. Fonte dos dados: NCEP (Silva et al., 2007 e Shi et al., 2000). que ocorreu precipitação intensa em parte do Estado de São Paulo, com precipitação acima de $100 \mathrm{~mm}$ próximo à Baixada Santista. Através do acompanhamento de imagens de satélite no canal infravermelho, nota-se a presença de um sistema frontal sobre o Estado de São Paulo no dia do evento. A configuração da nebulosidade apresenta uma situação tipo Y, em que há a presença de um sistema frontal e nebulosidade associada à Alta da Bolívia e ao vórtice ciclônico em altos níveis (VCAN) no nordeste brasileiro (Figura 2).

A configuração atmosférica para o dia do evento mostra uma região com um cavado em baixos níveis desde o Oceano Atlântico, adentrando o continente pela Região Sudeste e parte da Região Sul (Figura 3a-b) e a presença de confluência dos ventos em baixos níveis sobre o norte do Estado de São Paulo e sul de Minas Gerais (Figura 3b). Este padrão estava associado à atividade remanescente de um sistema frontal, como identificado na imagem de satélite (Figura 2). A configuração, em altos níveis, da Alta da Bolívia, do cavado na costa da Região Nordeste, de um cavado ao sul da região atingida e de uma área de difluência dos ventos próxima à mesma, sugere que esta região era propícia à convecção profunda (Figura $3 \mathrm{c}$ ). A região de estudo também apresentava convergência de umidade em baixos níveis e forte movimento ascendente na média troposfera, o que reforçava as condições conducentes para precipitação significativa (Figuras $3 \mathrm{~d}$-e, respectivamente). Apesar da grande escala favorecer o disparo de convecção úmida profunda sobre uma ampla área, sugere-se que fatores de mesoescala devem ter contribuído para modular a intensidade das chuvas, concentrando os maiores índices pluviométricos sobre uma pequena região. Por isto a importância de se empregar um modelo numérico de alta resolução, cujos resultados serão apresentados na próxima seção.

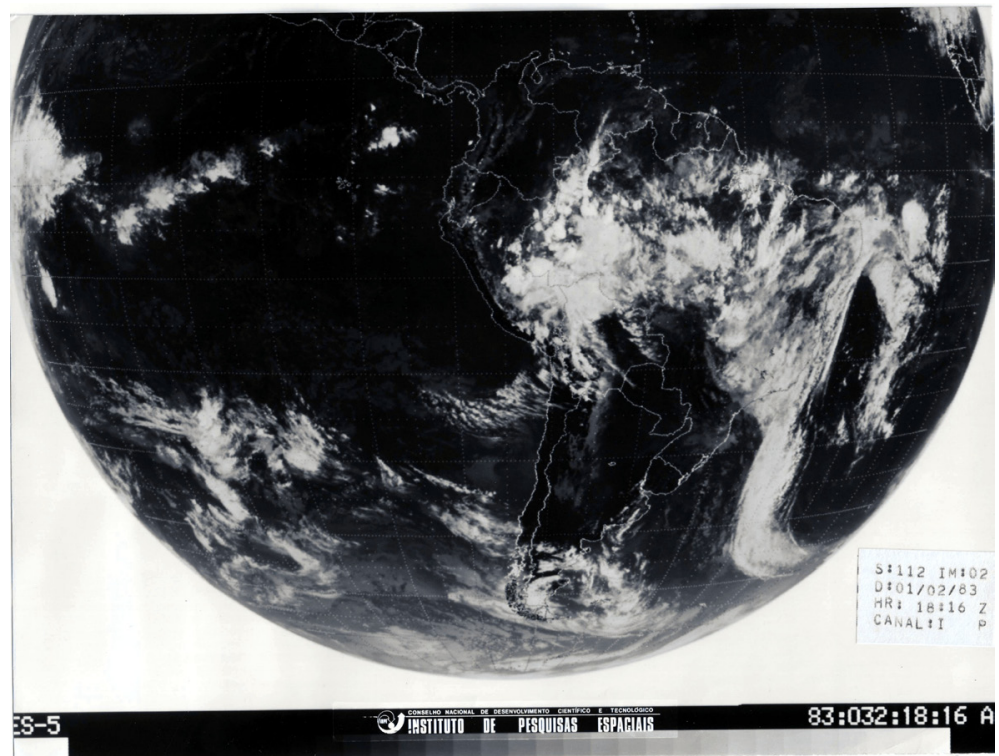

Figura 2 - Imagem do satélite GOES-5 no canal infravermelho para o dia 01/02/1983 18:16Z. 


\subsection{Análise da Simulação do Caso com o Modelo Regional Eta}

O máximo de precipitação acumulada em $24 \mathrm{~h}$ foi razoavelmente bem simulado pelo modelo, porém com um erro de fase no espaço. O núcleo de máxima precipitação simulada ficou deslocado para leste-nordeste, cobrindo o litoral norte de São Paulo e o litoral sul do Rio de Janeiro (Figura 4), enquanto que o máximo observado posicionou-se sobre a baixada santista (Figura 1). Para o litoral norte do Estado de São Paulo, os valores entre 60 e $80 \mathrm{~mm}$ concordaram com as observações. A simulação numérica gerou campos atmosféricos consistentes com a ocorrência de um episódio de altas taxas de precipitação na região estudada, apresentando os valores de temperatura mais altos e baixa pressão próxima dessa região (Figura 5a-b). Observa-se também, acompanhando a evolução da PNMM na região onde o evento foi simulado, uma queda no dia 01 às $18 \mathrm{~h}$ (Figura 6). A imagem de satélite apresentada

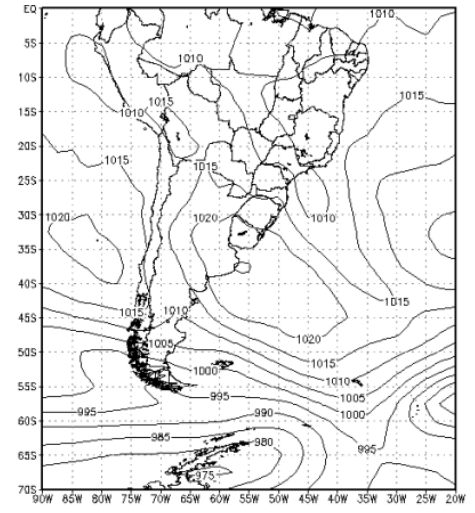

(a)

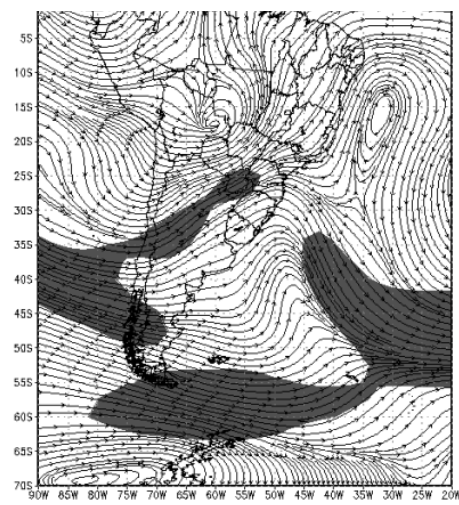

(c)

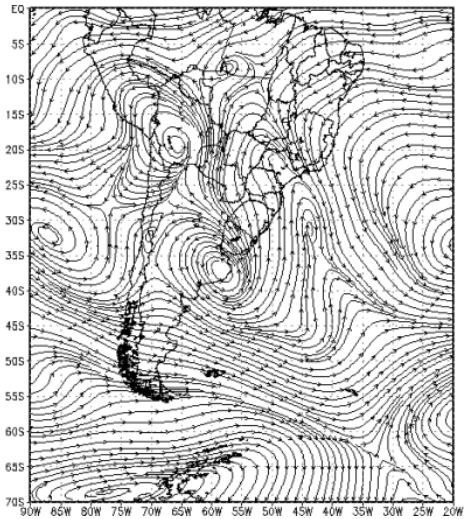

(b)

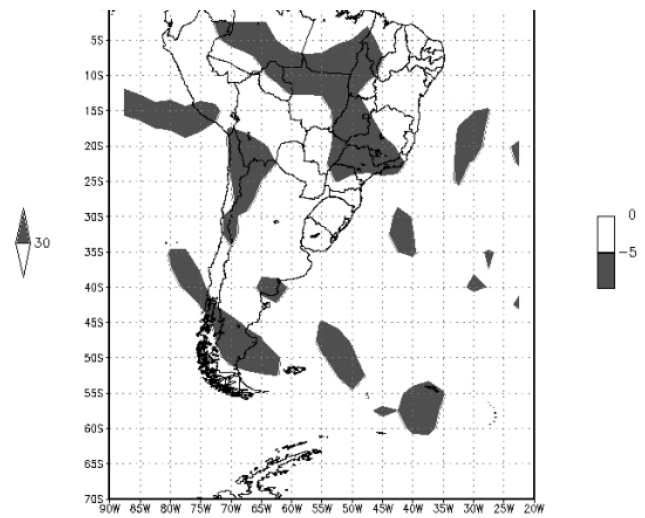

(d)

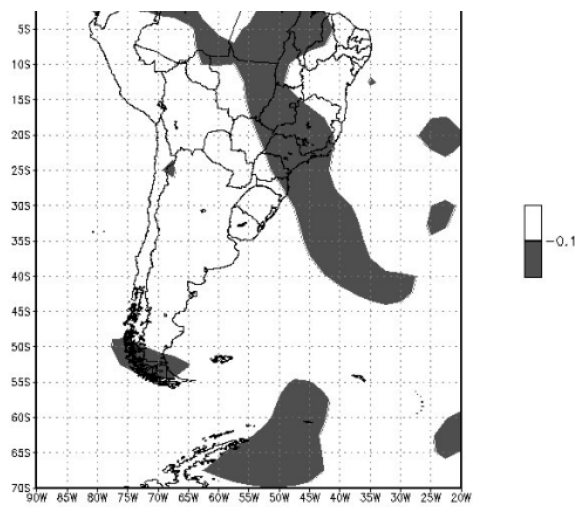

(e)

Figura 3 - Campos da Reanálise NCEP/NCAR para o dia 01/02/1983 18Z: a) PNMM (hPa); b) Linhas de corrente em 850 hPa; c) Linhas de corrente e magnitude do vento (m.s-1) em 250 hPa; d) Divergência de umidade (g.kg-1.s-1) em 850 hPa (E+08) e e) Omega (Pa.s-1) em 500 hPa. 


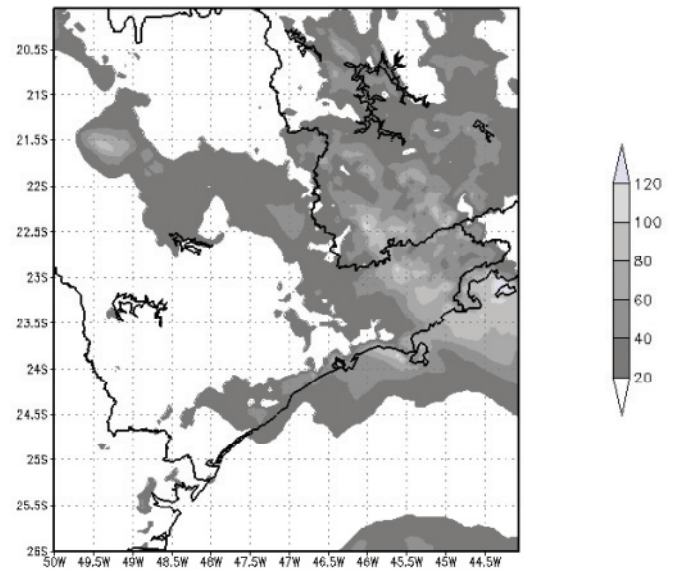

Figura 4 - Precipitação acumulada em 24h para o dia 02/02/1983 simulada pelo modelo Eta $5 \mathrm{Km}$. anteriormente (Figura 2), indicou que este foi o horário em que o evento estava acontecendo.

Para a análise dos perfis atmosféricos de estabilidade e umidade (e da convergência em baixos níveis), foram plotados os índices K, CK e CITT (Figura 7). Os índices CITT e CK foram plotados com a umidade em baixos níveis. Os índices K, CITT e CK mostraram resultados semelhantes, com valores bastante altos onde a chuva intensa foi simulada, indicando o potencial para tempestades geradoras de alto índice pluviométrico.

Ressalta-se que, no horário do evento (dia 01 às 1800Z), há uma confluência dos ventos e convergência de umidade em baixos níveis (Figura 5c e 5d) e movimento ascendente em baixos níveis (Figura 5e) sobre o litoral norte do Estado de São Paulo, região próxima da Serra do Mar. Com o fim de verificar a variabilidade diurna, analisamos, em conjunto com a evolução temporal da temperatura, a evolução temporal da
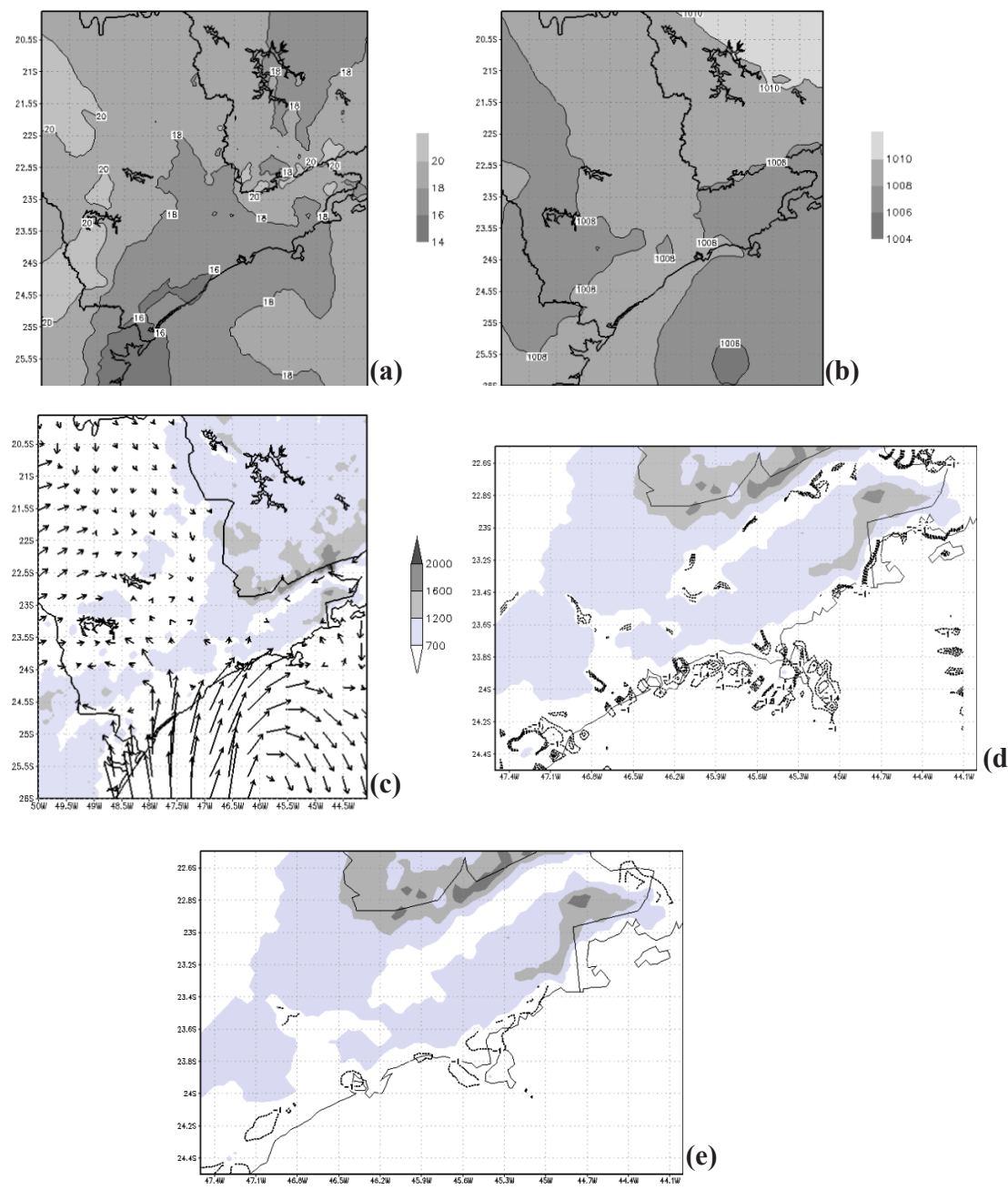

Figura 5 - Campos simulados pelo modelo Eta $5 \mathrm{Km}\left(01 / 02 / 1983\right.$ 18Z): a) Temperatura do ar $\left({ }^{\circ} \mathrm{C}\right)$ em $850 \mathrm{hPa}$; b) PNMM (hPa), c) Vento (m.s $\left.{ }^{-1}\right)$ em $925 \mathrm{hPa}$ e topografia $(\mathrm{m})$, d) Divergência de umidade $\left(\mathrm{g} \cdot \mathrm{kg}^{-1} \cdot \mathrm{s}^{-1}\right)$ em $925 \mathrm{hPa}(\mathrm{E}+04)$ e topografia $(\mathrm{m})$ e e) Omega $\left(\mathrm{Pa} \cdot \mathrm{s}^{-1}\right)$ em $850 \mathrm{hPa}$ e topografia $(\mathrm{m})$. 


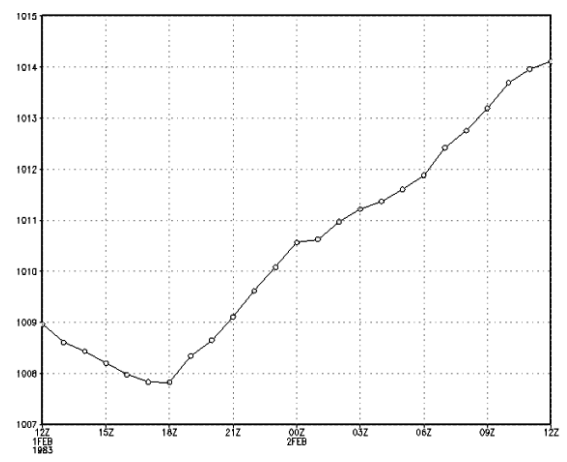

Figura 6 - Evolução temporal da PNMM (hPa) simulada pelo modelo Eta para a região do máximo de precipitação $\left(24^{\circ}-22^{\circ} \mathrm{S} ; 46^{\circ}-44^{\circ} \mathrm{W}\right)$.
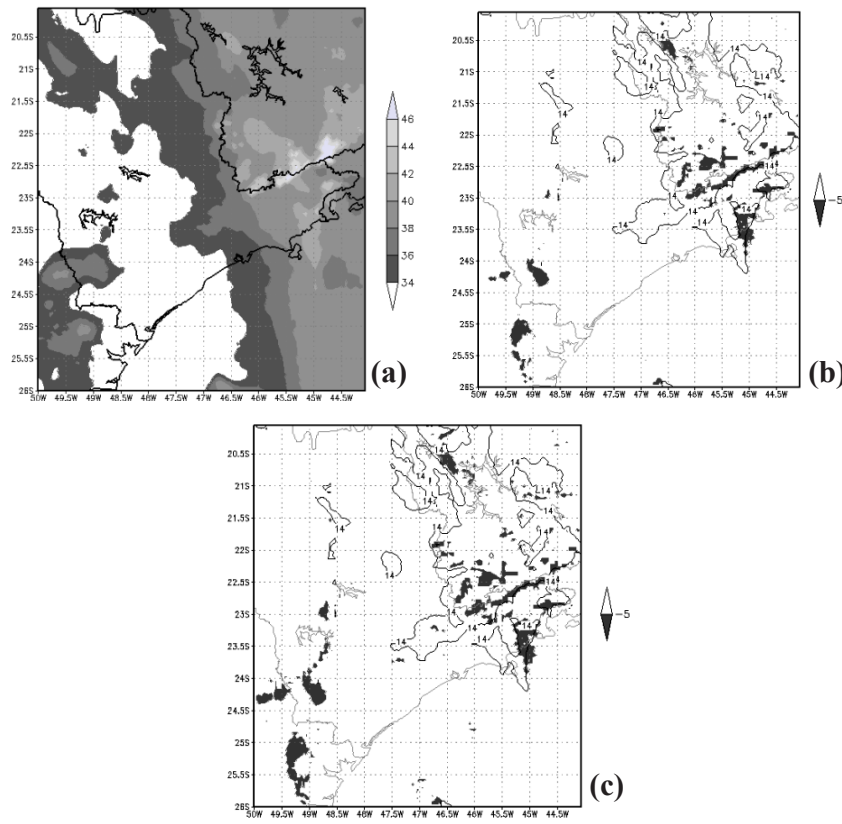

(b)

(c)

Figura 7 - Simulação do modelo Eta $5 \mathrm{Km}$ : (a) Índice $\mathrm{K}\left({ }^{\circ} \mathrm{C}\right)$ (01/02/1983 18Z); (b) Umidade (g.kg-1) em $850 \mathrm{hPa}$ (isolinhas) e índice CK ( $\left.{ }^{\circ} \mathrm{C} . \mathrm{s}-1\right)$ (sombreado) $(01 / 02 / 1983$ 18Z) e c) Umidade (g.kg-1) em $850 \mathrm{hPa}$ (isolinhas) e índice CITT ( $\left.{ }^{\circ} \mathrm{C} . s-1\right)$ (sombreado) (01/02/1983 18Z).

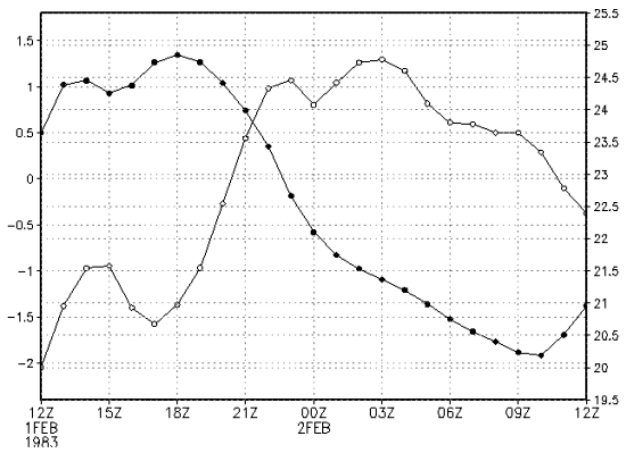

Figura 8 - Evolução temporal do desvio do vento zonal em relação à média do período de integração (diferença entre o vento zonal e a média do vento zonal) (m.s-1) (círculos vazios) e temperatura $\left({ }^{\circ} \mathrm{C}\right)$ (círculos cheios) em $925 \mathrm{hPa}$ simulado pelo modelo Eta para a região do máximo de precipitação $\left(24^{\circ}-22^{\circ} \mathrm{S} ; 46^{\circ}-44^{\circ} \mathrm{W}\right)$. diferença entre a componente zonal do vento e a média dessa componente em toda a integração do modelo na região onde ocorreu a máxima precipitação prevista (Figura 8). Pode-se observar um período diurno de ventos de leste (1200Z a 2000Z) e de oeste (2100 a 1000Z) de acordo com o ciclo de temperatura, que indicam a influência da brisa marítima no período do evento. Esses resultados sugerem que os efeitos conjuntos do vento de mesoescala (brisa) e do vento de escala sinótica (frente) na presença da orografia contribuíram para a formação do aglomerado convectivo naquela região.

\subsection{Caso 25 de Maio de 2005}

Este caso é de grande interesse devido à intensidade do fenômeno. Segundo o Jornal Vale Paraibano do dia 26 de maio de 2005, foram registrados no dia do evento a ocorrência de chuva forte e tornados, causando estragos em 14 cidades da região. O temporal destelhou 90 casas, provocou queda de dezenas de árvores, deixou 900 alunos sem aulas, afetou o abastecimento de energia elétrica e água e obstruiu rodovias da região.

A Figura 9 apresenta a precipitação acumulada entre os dias 24/05/2005 às $1200 \mathrm{Z}$ e 25/05/2005 às 1200Z. Observa-se que ocorreu precipitação intensa em grande parte do Estado de São Paulo, e valores bem altos sobre a região da Serra do Mar, onde a precipitação acumulada passou dos $100 \mathrm{~mm}$. Através do acompanhamento de imagens do satélite GOES -12 no canal

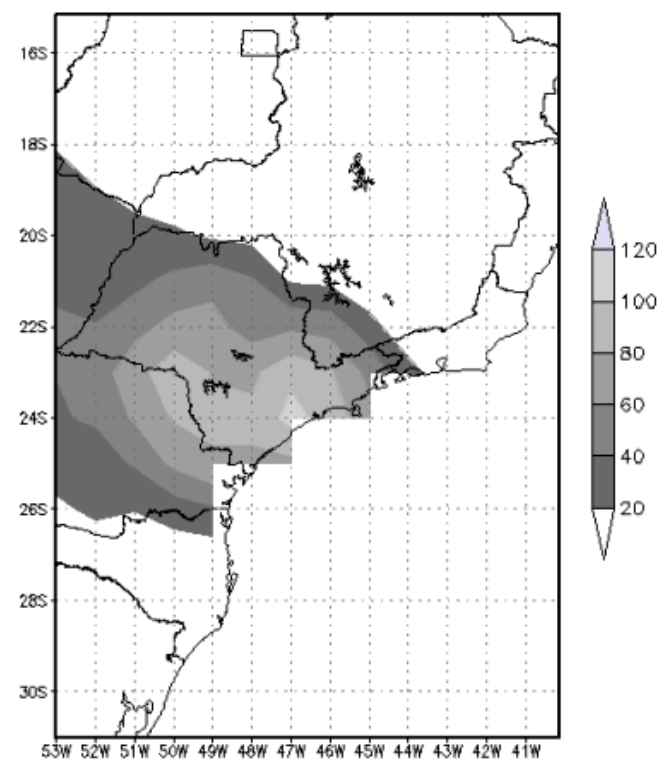

Figura 9 - Precipitação (mm) acumulada em 24h para o dia 25/05/2005. [Dados observados, em pontos de grade]. Fonte dos dados: NCEP (Silva et al., 2007 e Shi et al., 2000). 
infravermelho, nota-se a atuação de um sistema frontal passando pelo Estado de São Paulo no dia do evento, com presença de linhas de instabilidade (Figura 10a e 10c). Além disso, nota-se a presença de um aglomerado convectivo embebido no sistema frontal sobre parte da região da Serra do Mar (Figuras 10 b).

A configuração atmosférica para o dia do evento mostra uma região com pressão mais baixa desde o Oceano Atlântico, adentrando o continente pelo Estado de São Paulo e parte da Região Sul (Figura 11a). A configuração mostra também a presença de um escoamento em baixos níveis em uma faixa vindo desde a Amazônia até a Região Sudeste, e uma confluência dos ventos em baixos níveis sobre o Estado de São Paulo (Figura 11b). Essa confluência indica a atuação de um sistema frontal, consistente com as imagens de satélite. A região de estudo encontra-se também a leste do cavado em altitude, região esta propícia à convecção (Figura 11c), e na faixa onde ocorre a confluência em baixos níveis também há a presença de convergência de umidade em baixos níveis e movimento ascendente na média troposfera, o que reforça as condições para precipitação significativa (Figuras 11d-e, respectivamente). Esses resultados sugerem um escoamento de umidade vindo da região amazônica até o Sudeste. Entretanto, uma análise do perfil vertical do vento indicou a não existência da configuração de um jato em baixos níveis (não mostrado). Observa-se também uma faixa de vorticidade negativa e positiva associadas ao cavado e crista do escoamento, acompanhando o sistema frontal
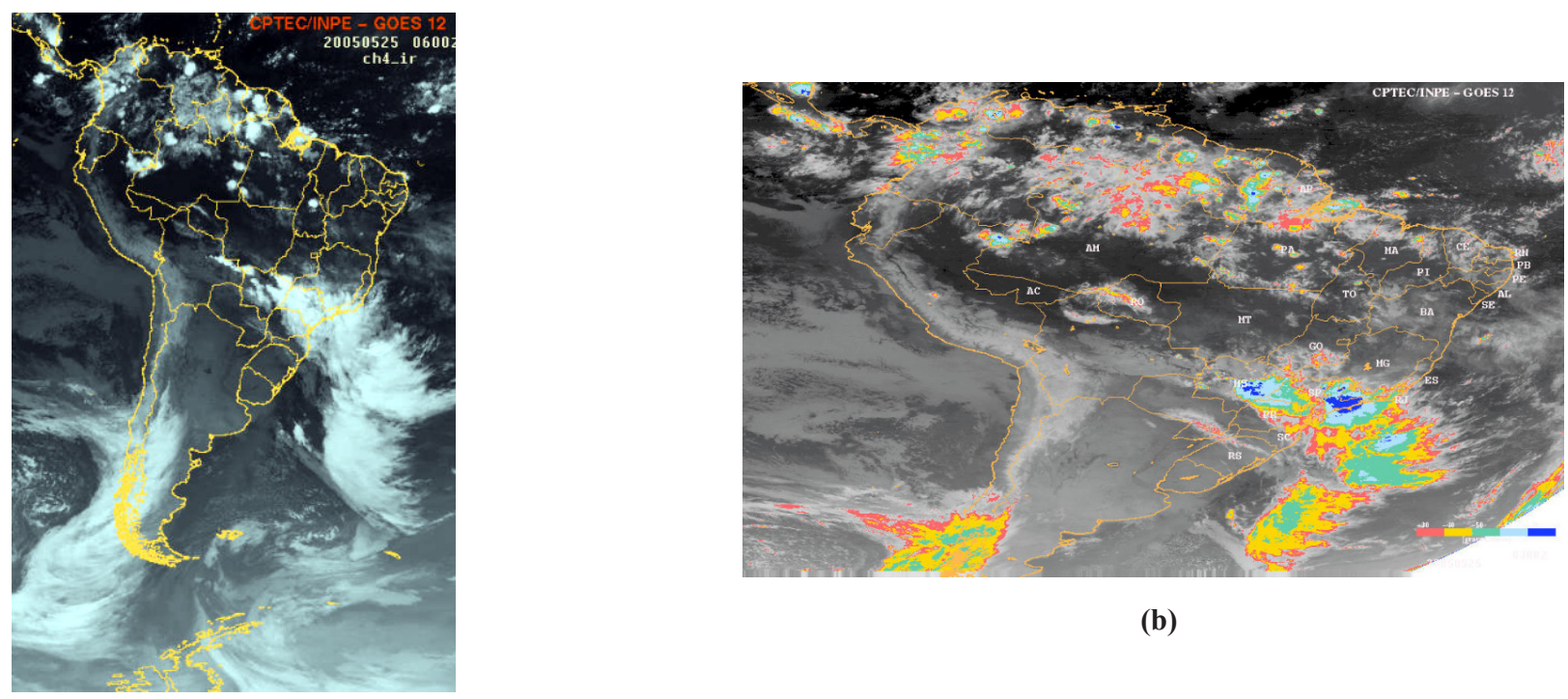

(b)

(a)

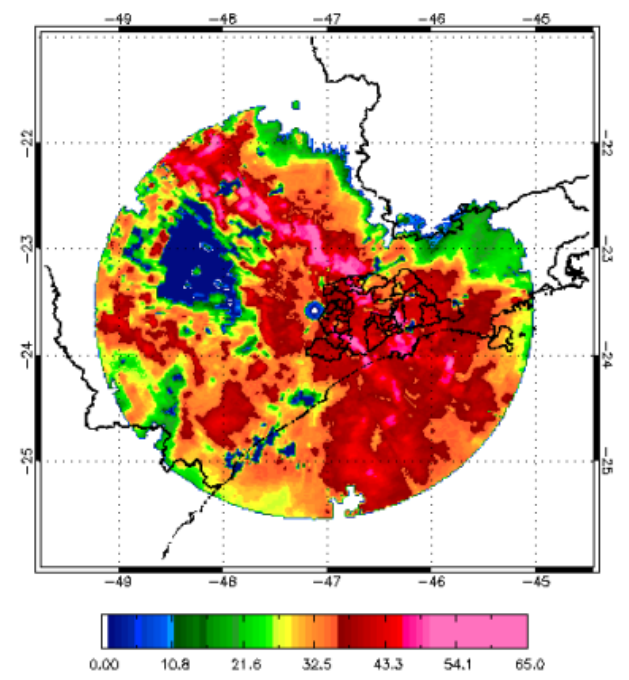

(c)

Figura 10 - Imagens de satélite do GOES-12: IV (25/05/2005 06Z) (a) e IV realçada (25/05/2005 02:54Z) (b), e Imagem do radar de São Roque CAPPI 3 Km (dbz) (25/05/2005 02Z) (c).

[Fonte: CPTEC/INPE] 


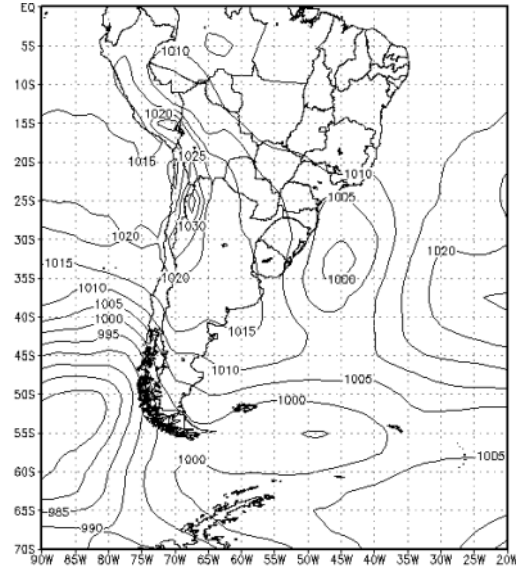

(a)

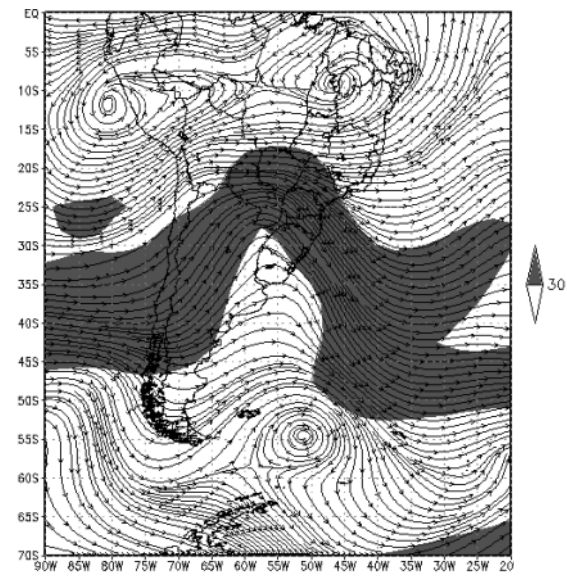

(c)

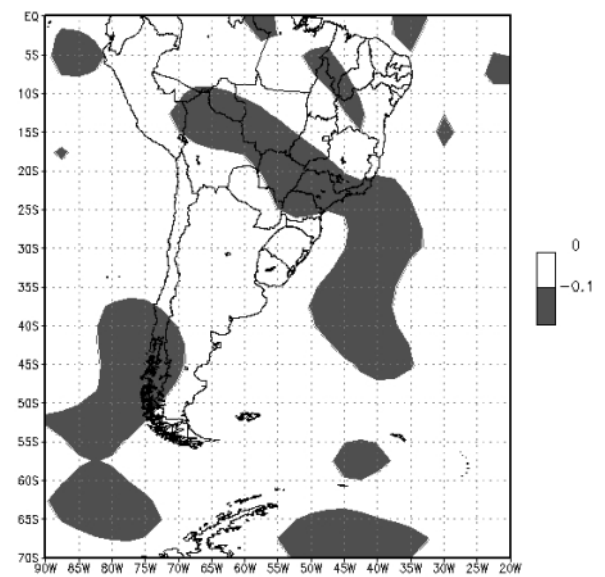

(e)

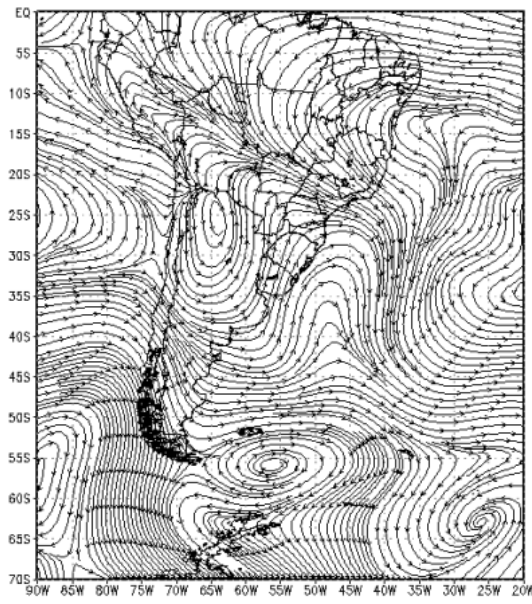

(b)

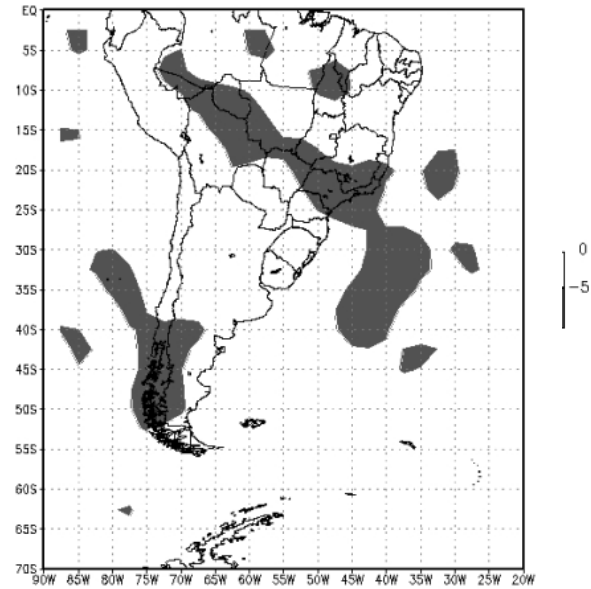

(d)

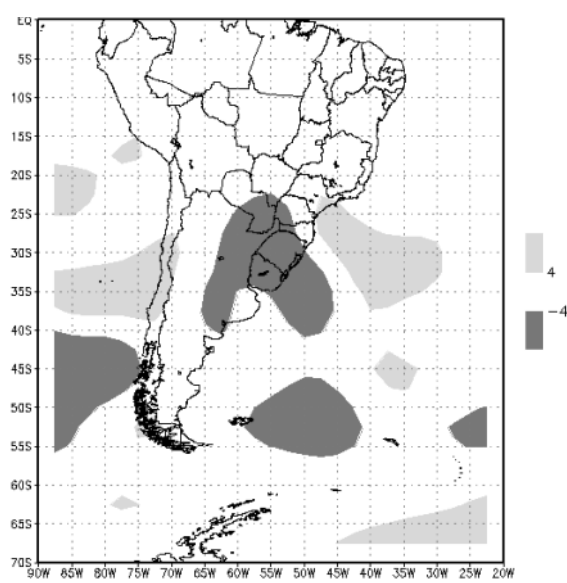

(f)

Figura 11 - Campos da Reanálise NCEP/NCAR para o dia 25/05/2005 06Z: PNMM (hPa) (a), Linhas de corrente em 850 hPa (b), Linhas de corrente e magnitude do vento (m.s-1) em $250 \mathrm{hPa}$ (c), Divergência de umidade (g.kg-1.s-1) em 850 hPa (E+08) (d), Omega (Pa.s-1) em 500 hPa (e) e Vorticidade relativa (s-1) em $250 \mathrm{hPa}$ (f) 
(Figura 11f). Todos esses resultados indicam a atuação de um sistema frontal, com condições atmosféricas propícias para forte precipitação na região de estudo. Entretanto, a região que apresenta as configurações acima é bastante extensa e por isso é necessário avaliar mais detalhadamente para verificar quais outros fatores contribuíram para a ocorrência de precipitação intensa somente na região próxima à Serra do Mar. Para isto, foi utilizado o modelo regional Eta, com o objetivo de verificar a configuração atmosférica de mesoescala para a região onde ocorreu a precipitação intensa, além da verificação da previsão.

\subsection{Análise da Simulação do Caso com o Modelo Regional Eta}

O máximo de precipitação acumulada em $24 \mathrm{~h}$ na região da Serra do Mar foi relativamente bem representado pelo modelo Eta-5 Km. Porém, o núcleo de precipitação máxima simulada ficou deslocado mais para sul, quando comparado com as observações (Figura 12). Entretanto, o modelo simulou bem a presença do sistema frontal, com a banda convectiva se estendendo do continente até o oceano, como notado nas imagens de satélite. O modelo também conseguiu simular bem as características sinóticas associadas ao sistema, apresentando, na região em estudo, um gradiente de temperatura (Figura 13a), baixa pressão (Figura 13b), confluência dos ventos em baixos níveis (Figura 13c).

Para a análise dos perfis atmosféricos de estabilidade e umidade (e da convergência em baixos níveis), foram plotados os índices K, CK e CITT (Figura 14). Os índices CITT e CK foram plotados juntamente com a umidade em baixos níveis. $\mathrm{O}$ índice $\mathrm{K}$ mostrou uma extensa área favorável à tempestade, incluindo a região de estudo (Figura 14a). Tanto o índice CITT quanto o CK (Figura 14b-c, respectivamente), juntamente com altos valores de umidade, mostraram que a região onde ocorreu o máximo de precipitação apresentava-se favorável à formação de tempestades.

Analisando o vento em baixos níveis (Figura 13c), observa-se que a região de encontro entre a faixa de confluência dos ventos e a região a barlavento da orografia da Serra do Mar é a localização do núcleo previsto de precipitação (Figura 12). O regime de brisa não afeta significativamente esse núcleo de precipitação, uma vez que, além deste estar a barlavento da Serra do Mar, a máxima precipitação ocorreu durante a manhã, onde a brisa ocorre do continente para o mar (brisa terrestre). Observa-se também nesta área uma convergência de umidade e movimento ascendente em baixos níveis (Figura 13d-e). Esses resultados sugerem que a presença da orografia foi o fator de mesoescala que provocou esse máximo de precipitação localizado.
Observa-se que os valores de omega simulados pelo modelo, em ambos os casos, apresentam uma ordem de grandeza maior que o apresentado pela Reanálise. Isso se deve ao fato da resolução do modelo ser bem maior, fazendo com que ele represente também o movimento ascendente devido à mesoescala, enquanto a Reanálise somente representa o movimento ascendente devido à escala sinótica.

\section{CONSIDERAÇÕES FINAIS}

Os dois casos estudados neste capítulo apresentaram precipitação acima de $100 \mathrm{~mm} /$ dia na região próxima à Serra do Mar - 02/02/1983 e 25/05/2005. O primeiro ocorreu durante um verão considerado chuvoso e o segundo durante um outono também considerado chuvoso. Em ambos os casos houve ocorrência de um aglomerado convectivo embebido em um sistema frontal. No primeiro caso a situação sinótica possuía características de um sistema tipo $\mathrm{Y}$, onde há presença de nebulosidade em que se pode identificar o sistema frontal, a presença da Alta da Bolívia e do VCAN próximo ao nordeste brasileiro. No segundo caso havia presença de linha de instabilidade além dos aglomerados convectivos embebidos no sistema frontal.

O máximo de precipitação acumulada em $24 \mathrm{~h}$ foi bem simulado pelo modelo nos dois casos. Porém no primeiro, o máximo de precipitação foi deslocado para o norte, alcançando o litoral norte de São Paulo e o litoral sul do Rio de Janeiro. No segundo caso, a precipitação simulada se concentrou em uma faixa noroeste-sudeste que não atingiu todo o Estado de São Paulo. O modelo também simulou bem as características sinóticas, tais como a confluência dos ventos, gradiente de

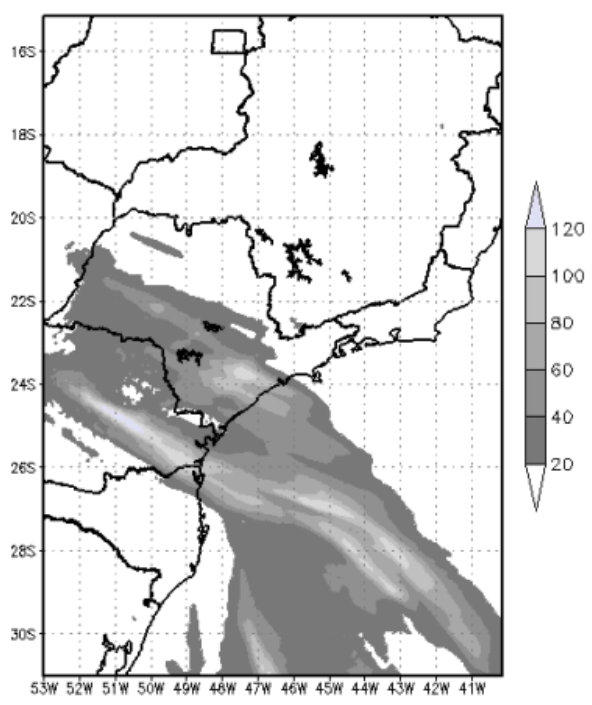

Figura 12 - Precipitação (mm) acumulada em 24h para o dia 25/05/2005 simulada pelo modelo Eta $10 \mathrm{Km}$. 


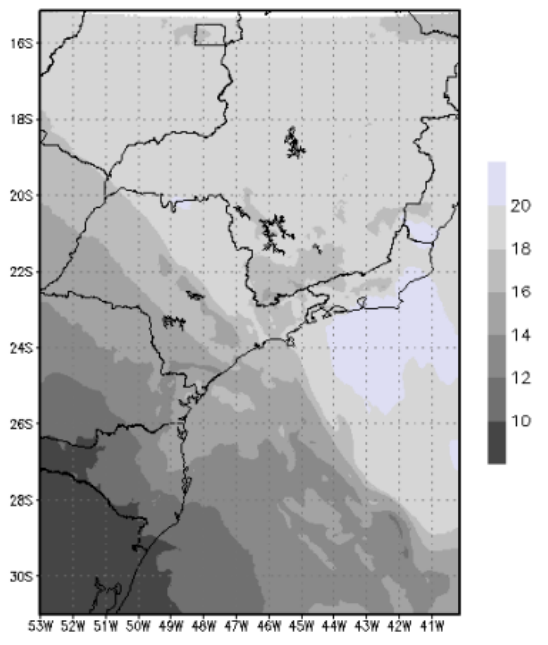

(a)

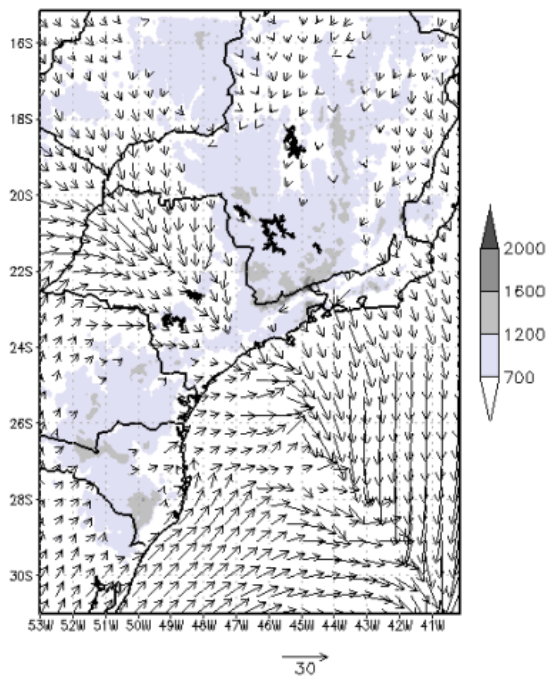

(c)

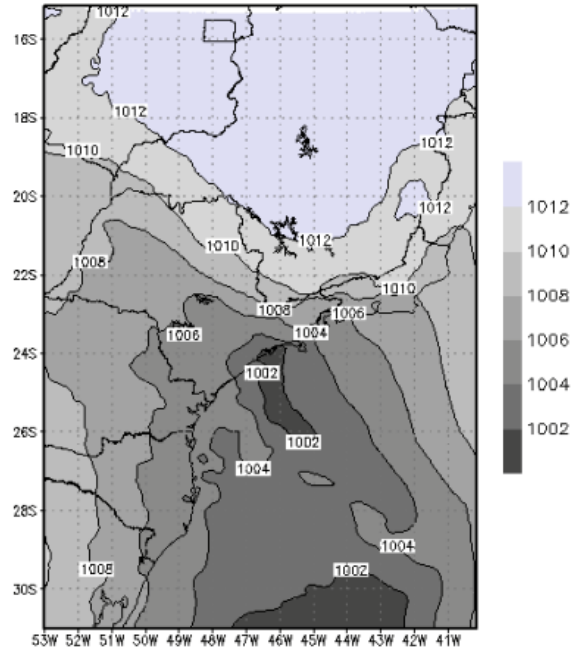

(b)

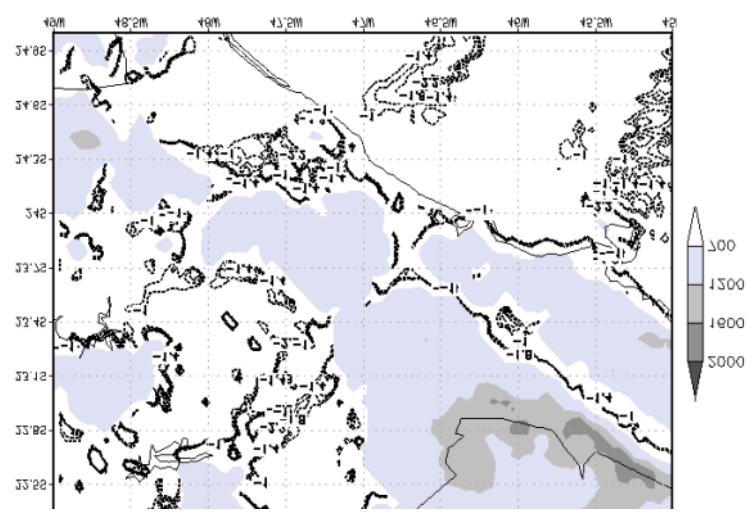

(d)

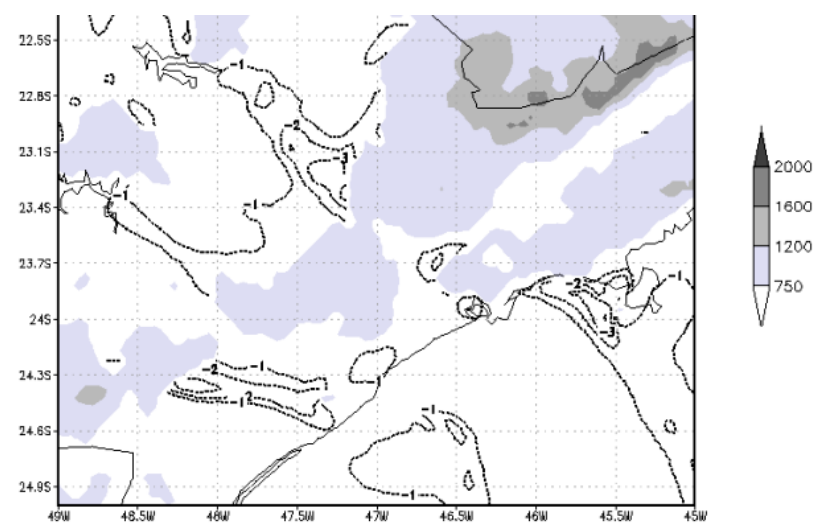

(e)

Figura 13 - (a) Temperatura do $\operatorname{ar}\left({ }^{\circ} \mathrm{C}\right)$ em 850 hPa (25/05/2005 09Z), (b) PNMM (hPa) (25/05/2005 06Z), (c ) vento (m.s-1) em 925 hPa e topografia (25/05/2005 08Z), (d) Divergência de umidade (g.kg-1.s-1) em 925 hPa (E+04) e topografia (m) (25/05/2005 09Z), (e) Divergência de umidade (g.kg-1.s-1) em 925 hPa (E+04) e topografia (m) (25/05/2005 09Z) ) 


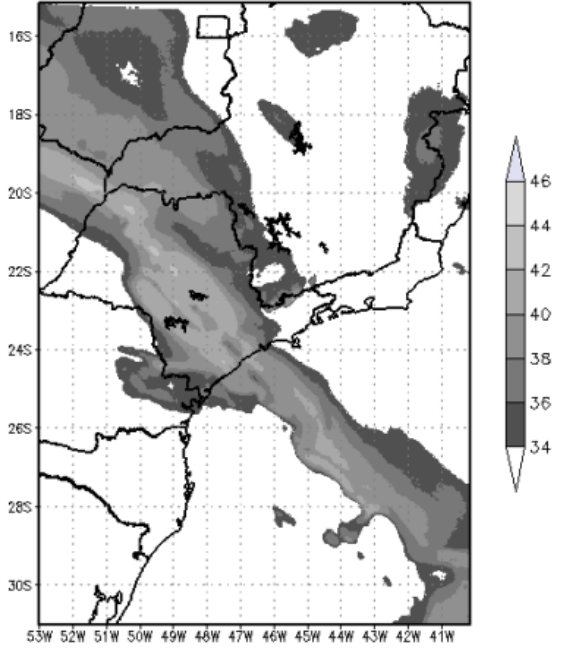

(a).

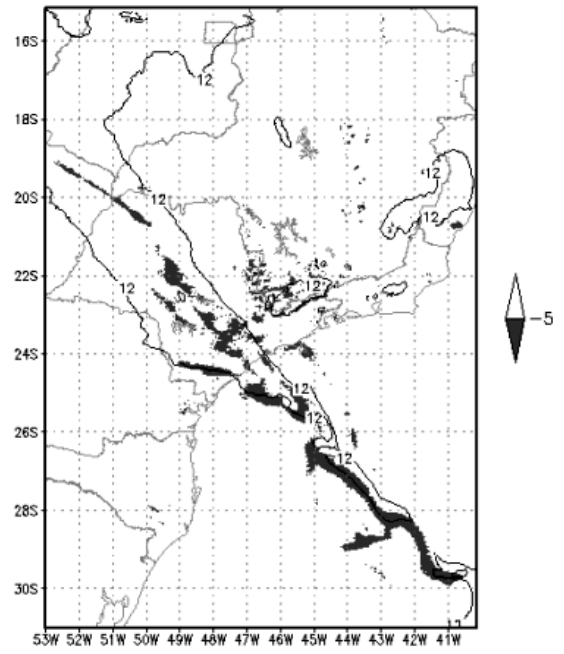

(b)

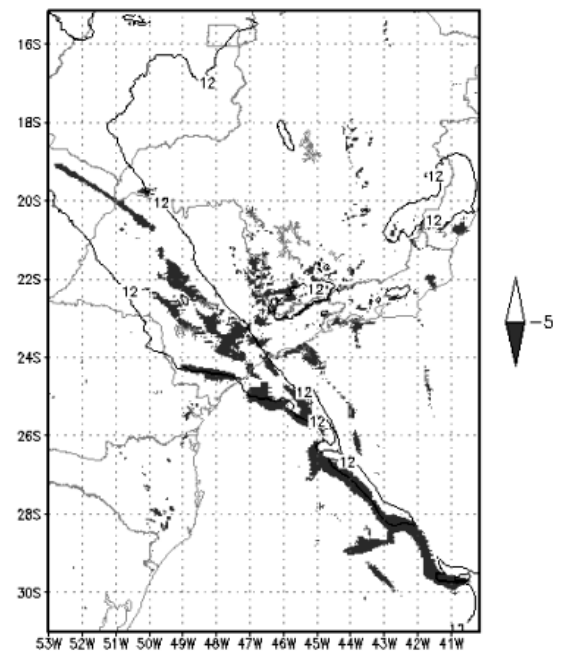

Figura 14 - Simulação do modelo Eta $10 \mathrm{Km}$ : (a) Índice $\mathrm{K}\left({ }^{\circ} \mathrm{C}\right)$ (25/05/2005 08Z), (b) Umidade (g.kg-1) em $850 \mathrm{hPa}$ (isolinhas) e índice CK $\left({ }^{\circ}\right.$ C.s-1) (sombreado) $(25 / 05 / 200509 Z)$ e (c) Umidade (g.kg-1) em $850 \mathrm{hPa}$ (isolinhas) e índice CITT $\left({ }^{\circ} \mathrm{C} . s-1\right)$ (sombreado) $(25 / 05 / 2005$ 09Z). temperatura, pressões baixas, presença de cavado em altos níveis, acompanhando os sistemas frontais. Os índices de instabilidade utilizados representaram bem a instabilidade atmosférica. As características de mesoescala também foram bem representadas, com relação aos indícios da ação da brisa marítima (primeiro caso) e orografia (primeiro e segundo caso), os quais podem ter contribuído para a intensidade dos eventos. Para entender melhor o papel da orografia nesses eventos de chuva intensa seria interessante, em um estudo posterior, realizar a análise de simulações com o Modelo Regional Eta retirando a topografia.

\section{AGRADECIMENTOS}

Ao Projeto Serra do Mar apoiado pela FAPESP (04/09649-0) e ao CNPq (PQ 308725/2007-7, 307913/2007-4 e AT 373194/2006-4).

\section{REFERÊNCIAS BIBLIOGRÁFICAS}

\section{CERQueirA, F. A. Avaliação do Modelo Eta durante} episódios de ZCAS. 2006. Monografia (Bacharel em Meteorologia) - Universidade Federal do Rio de Janeiro (UFRJ), Rio de Janeiro, 2006.

CHOU, S. C. Modelo regional ETA. Climanálise - Boletim de Monitoramento e Análise Climática, v. 1, n. especial 10 anos, 1996. (INPE-12512-PRE/7814). Disponível em: <http://tucupi.cptec.inpe.br/products/climanalise/ cliesp10a/27.html>. Acesso em: 17 abr. 2009.

GUIMARÃES, J. M.; CHAN, C. S.; DERECZYNSKI, C. P. Avaliação do modelo ETA para um caso de chuvas intensas entre os estados do Rio de Janeiro e do Espírito Santo. In: SEMINÁRIO DE INICIAÇÃO CIENTÍFICA (SICINPE), 2007, São José dos Campos-SP. Resumos... São José dos Campos-SP: INPE, 2007. Disponível em: <http://urlib.net/ sid.inpe.br/mtc-m17@80/2007/12.17.13.18>. Acesso em: 17 abr. 2009.

KAIN J. S.; FRITSCH, J. M. Convective parameterization for mesoscale models: The Kain-Fritsch scheme. The Representation of Cumulus Convection in Numerical Models, Meteor. Monogr., n. 24, p. 165-170, 1993.

KALNAY, E. et al. The NCEP/NCAR 40-Year Reanalysis Project. Bull. Amer. Meteorol. Soc., v. 77, n. 3, p. 437-471, 1996.

LIMA, D. R. O. Diagnóstico de chuvas e previsão meteorológica para a bacia hidrográfica do rio Manso. 2005. Dissertação (Mestrado em Engenharia Civil) - Universidade Federal do Rio de Janeiro (COPPE/UFRJ), Rio de Janeiro, 2005.

MESINGER, F. A blocking technique for representation of montains in atmospheric model. Riv. Meteor. Aeronaut., v. 44, n. 1-4, p. 195-202, 1984. 
MESINGER, F.; JANJIC, Z. I.; NICKOVIC, S.; GAVRILOV, D.; DEAVEN, E. D. G. The step-mountain coordinate: model description and performance for cases of Alpine lee cyclogenesis and for a case of Appalachian redevelopment. Mon. Wea. Rev., v. 116, n. 7, p. 1493-1518, 1988.

ROZANTE, J. R.; CAVALCANTI, I. F. A. Influência da condição inicial na simulação do evento de precipitação extrema na serra do mar. In: CONGRESSO BRASILEIRO DE METEORologia (CBMET), XIV, 2006, Florianópolis, SC. Anais... Florianópolis: SBMET, 2006. Disponível em: $<$ http://urlib.net/sid.inpe.br/mtcm17@80/2006/12.21.18.25>. Acesso em: 17 abr. 2007.

SHI, W.; HIGGINS, R. W.; YAROSH, E.; KOUSKY, V. E. The annual cycle and variability of precipitation in Brazil. NCEP/Climate Prediction Center ATLAS no 9, 2000. Disponível em: < http://www.cpc.ncep.noaa.gov/ research_papers/ncep_cpc_atlas/9/toc.html > Acesso em: 17 abr. 2009.
SILVA, V. B. S.; KOUSKY, V. E.; SHI, W.; HIGGINS, R. W. An improved gridded historical daily precipitation analysis for Brazil. J. Hydrometeorol., v. 8, n. 4, p. 847-861, 2007. VASCONCELLOS, F. C. Variabilidade atmosférica associada a casos extremos de precipitação na Região Sudeste do Brasil. 2008. Dissertação (Mestrado em Meteorologia) Instituto Nacional de Pesquisas Espaciais, São José dos Campos, 2008. Disponível em: <http://urlib.net/sid.inpe.br/ mtc-m17@80/2008/01.15.16.05>.Acesso em: 17 jul.2009. 